\title{
Magnetic resonance relaxometry improves the accuracy of conventional MRI in the diagnosis of endometriosis-associated ovarian cancer: A case report
}

\author{
SHO MATSUBARA ${ }^{1}$, NAOKI KAWAHARA ${ }^{1}$, AKIHITO HORIE ${ }^{2}$, RYUSUKE MURAKAMI ${ }^{2}$, \\ NAOKI HORIKAWA $^{2}$, DAICHI SUMIDA ${ }^{1}$, TAKUYA WADA ${ }^{1}$, TOMOKA MAEHANA ${ }^{1}$, AIKA YAMAWAKI ${ }^{1}$, \\ MAYUKO ICHIKAWA $^{1}$, CHIHARU YOSHIMOTO ${ }^{1}$, MASAKI MANDAI ${ }^{2}$ and HIROSHI KOBAYASHI ${ }^{1}$ \\ ${ }^{1}$ Department of Obstetrics and Gynecology, Nara Medical University, Kashihara, Nara 634-8522; \\ ${ }^{2}$ Department of Obstetrics and Gynecology, Kyoto University, Kyoto 606-8507, Japan
}

Received December 10, 2018; Accepted June 14, 2019

DOI: $10.3892 / \mathrm{mco} .2019 .1889$

\begin{abstract}
Endometriosis is a precancerous condition for endometriosis-associated ovarian cancer (EAOC). In the present study, conventional magnetic resonance imaging (MRI) and MR relaxometry were used to examine a case of clear cell carcinoma that arose in a pre-existing right-sided benign ovarian endometrioma (OE). The 42-year-old nulliparous woman suspected of EOAC, as assessed by conventional MRI, requested fertility-sparing surgery such as laparoscopic endometrioma cystectomy. Furthermore, the MR transverse relaxation rate (R2) was determined using a single-voxel, multi-echo MR sequence using a 3 Tesla-MR system. An R2 value $<12.1 \mathrm{~s}^{-1}$ was indicative of malignancy, as described in previous studies. In the present study, MR relaxometry identified an R2 value of $7.98 \mathrm{~s}^{-1}$ in the right cyst, which suggested the malignant transformation of benign OE. Based on these findings, fertility-sparing surgery was contraindicated. In conclusion, MR relaxometry may represent a new clinical approach as an adjunctive modality for the diagnosis of EAOC. When patients exhibiting a pelvic mass suspected of EAOC desire fertility-sparing treatment options, MR relaxometry can facilitate the selection of conservative management.
\end{abstract}

Correspondence to: Dr Sho Matsubara, Department of Obstetrics and Gynecology, Nara Medical University, 840 Shijo-cho, Kashihara, Nara 634-8522, Japan

E-mail: rsa63163@naramed-u.ac.jp

Abbreviations: EAOC, endometriosis-associated ovarian cancer; OE, ovarian endometrioma; MR, magnetic resonance; MRI, MR imaging; T1WI, T1-weighted image; T2WI, T2-weighted image; CA, carbohydrate antigen

Key words: endometriosis, malignant transformation, MRI, MR relaxometry

\section{Introduction}

Endometriosis is defined as the presence of endometrial glands and stroma outside the uterine cavity. Endometriosis is a common disease affecting $10 \%$ of women of reproductive age. The lesions are mainly located in the ovaries, peritoneal cavity, recto-uterine pouch and extrapelvic areas. Malignant transformation is a rare complication of endometriosis, occurring in $0.6-0.8 \%$ of all cases (1). Clinical characteristics of endometriosis-associated ovarian cancer (EAOC) are frequently observed in ovarian endometrioma (OE). Ovarian clear cell carcinoma is the most frequent form of EAOC in Japan, followed by endometrioid carcinoma (2). Ultrasound remains the initial and most useful imaging method for the detection of ovarian cancer, while magnetic resonance imaging (MRI) is the second-line imaging modality. However, MRI is the most optimal imaging method to distinguish between benign and malignant ovarian cystic lesions (3). MR criteria for the diagnosis of EAOC include the presence of a large ovarian cyst with hemorrhagic fluid, various solid portions, mural nodules, papillary projections, thick septations or significant vascularization, as assessed by contrast medium administration. The features of imaging modalities, including dynamic contrast-enhanced imaging, high signal intensity of diffusion-weighted images and low apparent diffusion coefficient values facilitate the diagnosis of EAOC (4). To the best of our knowledge, the only effective treatment to treat EAO consists in the extensive excision of the tumor.

In addition to oncological outcomes, the management of nulliparous women who have high risk of EAOC represents a challenge, due to the potential impact of EAOC on their fertility. When the patients select fertility-preserving alternatives, surgeon must carefully consider each indication (5). In general, MRI can facilitate the correct preoperative characterization of the lesion. However, in a study by Tanase et al (4), it was shown that a part of the benign mass can exhibit increased mural nodules and solid components. For at-risk populations, MR relaxometry is an emerging radiological technique that may improve the prediction of EAOC occurrence (6). In a study by Yoshimoto et al (6) transverse relaxation rate (R2) was determined using a single-voxel, multi-echo MR sequence 
(HISTO) using a 3 Tesla (3T)-MR system, and it was reported that preoperative MR relaxometry is a useful tool for distinguishing EAOC from benign $\mathrm{OE}$.

The present study reported the case of a 42-year-old nulliparous woman who was suspected of EAOC but desired a fertility-sparing surgery, such as laparoscopic endometrioma cystectomy. MR relaxometry was performed, and the MR relaxometry results confirmed the diagnosis of EAOC.

\section{Case presentation}

The patient was a 42-year-old nulliparous woman with a history of $\mathrm{OE}$ that occurred 4 years prior to admission to our hospital. She presented mild cyclic pelvic pain, which required admission to another clinic. She underwent hysteroscopic resection of benign endometrial polyps 3 years prior to admission to our hospital. The patient was admitted to the Nara Medical University Hospital in April 2017 for the analysis of a complex bilateral adnexal mass identified by sonographic examination. Physical examination indicated right adnexal tenderness. The carbohydrate antigen (CA) 125 and CA 19-9 levels at presentation were $50.4 \mathrm{U} / \mathrm{ml}$ and $26.1 \mathrm{U} / \mathrm{ml}$, respectively. Transvaginal ultrasonography, which was performed in Kyoto University Hospital in May 2017, identified a multilocular cystic mass with a size of $\sim 64 \times 52 \mathrm{~mm}$ in the right ovary (Fig. 1), and one cystic mass with a size of $\sim 42 \times 24 \mathrm{~mm}$ in the left ovary, accompanied with solid mural nodules in the right adnexa with diameters of $12 \mathrm{~mm}$. The present findings were consistent with those of bilateral OE. The patient underwent routine MRI using T1-weighted (T1W) and T2-weighted (T2W) sequences. The tumor exhibited areas of high signal intensity using both T1W imaging (T1WI) and T2WI, suggestive of subacute intra-cystic hemorrhage (Fig. 2). MR images of the pelvis revealed bilateral multilocular cystic masses suggestive of mural nodules, which showed heterogeneous enhancement on the post-contrast fat-saturated T1WI, due to the increased vascularity in the right adnexal mass (Fig. 3). Collectively, these features were suggestive of a malignant transformation of endometriosis.

The patient desired to preserve her fertility despite the oncological hazards. A surgery that included staging, right salpingo-oophorectomy, left cystectomy, omentectomy, peritoneal washings and biopsies were suggested in order to perform an accurate diagnosis, followed by retroperitoneal lymph node dissection. However, the patient refused this treatment. Gynecologic oncologists and radiologists advised the patient to undergo MR relaxometry (MAGNETOM Skyra; 3T MRI; Siemens Healthcare $\mathrm{GmbH}$ ) of the pelvis. After routine MRI, the patient underwent MR relaxometry using a single-voxel spectroscopy, a stimulated echo acquisition mode sequence at multiple echoes, and fitting of an exponential decay to the amplitude at each echo (6). The $\mathrm{R} 2$ value parameter was calculated using a high-speed T2*-corrected HISTO using a 3T-MR system, as previously described (6). MR relaxometry identified an R2 value of $7.98 \mathrm{~s}^{-1}$ in the right cyst, which suggested a malignant transformation of OE (Fig. 4). Malignant potential was identified by MRI only on the right side, and evaluation with MR relaxometory on the left ovary was not performed because malignant potential was not identified by MRI. Following MRI and MR relaxometry, EAOC was pre-operatively diagnosed. Computed tomography (CT) scan was performed in the previous hospital, and no metastasis was identified. Based on these findings, fertility-sparing surgery was contraindicated in this patient. She underwent right salpingo-oophorectomy, left cystectomy, omentectomy, peritoneal biopsies and retroperitoneal lymphadenectomy. The histopathological results identified clear cell carcinoma arising from endometriosis (Fig. 5) at International Federation of Gynecology and Obstetrics stage IC1 (7). The carcinoma did not extend to the solid components within the cyst and remained limited to non-solid regions in the cyst. Peritoneal washing results were negative for malignant cells. Adjuvant chemotherapy was recommended, but the patient refused it. The patient was asymptomatic without recurrence or metastasis at the 2-year follow-up examination, which was performed at another hospital.

Immunohistochemistry. Tissues were fixed with $10 \%$ neutral buffered formalin-fixed at $20^{\circ} \mathrm{C}$ for $24 \mathrm{~h}$, and paraffin-embedded tissues were cut into $4-\mu \mathrm{m}$-thick sections. The slides were deparaffinized in xylene at $20^{\circ} \mathrm{C}$ for $30 \mathrm{~min}$ and rehydrated in a graded alcohol series. Endogenous peroxidase was inactivated with $3 \%$ hydrogen peroxide at $20^{\circ} \mathrm{C}$ for $10 \mathrm{~min}$. Tissue sections were incubated in $0.1 \mathrm{M}$ citrate buffer $(\mathrm{pH}$ 6.0) and placed in an autoclave during antigen retrieval at $125^{\circ} \mathrm{C}$ for $5 \mathrm{~min}$. The sections were blocked with $1 \%$ BSA diluted in PBS at $20^{\circ} \mathrm{C}$ for $10 \mathrm{~min}$, and then incubated with a primary antibody anti-hepatocyte nuclear factor- $1 \beta$ (1:500; Sigma-Aldrich; Merck; cat. no. MABE971) at $4^{\circ} \mathrm{C}$ overnight and incubated with secondary antibody hydrogen peroxidase-conjugated (1:150; Sigma-Aldrich; Merck; cat. no. A9917) at $20^{\circ} \mathrm{C}$ for $60 \mathrm{~min}$. Sections were subjected to the peroxidase reaction using $0.02 \% 3,3$ '-diaminobenzidine tetrahydrochloride at $20^{\circ} \mathrm{C}$ for $10 \mathrm{~min}$, followed by counterstaining with hematoxylin at $20^{\circ} \mathrm{C}$ for $5 \mathrm{~min}$. Sections were visualized using a light microscope (magnification, x200).

\section{Discussion}

The present case study suggested that preoperative MR relaxometry may be a useful tool for distinguishing EAOC from benign $\mathrm{OE}$. The $\mathrm{R} 2$ value could guide patient selection prior to conservative management, including fertility-sparing surgery, and may serve as an effective parameter for the diagnosis of EAOC.

MRI has high accuracy in differentiating between benign and malignant endometriosis (4). Malignant transformation is a rare complication of endometriosis, affecting $0.6-0.8 \%$ of all cases of OE (1). In Japan, clear cell carcinoma is not uncommon and is the second most frequently occurring type of EAOC, accounting for $>20 \%$ of all EAOC cases (8). In the present case report, transvaginal ultrasonographic findings revealed a large pelvic mass and solid echogenic loculi. Furthermore, based on the MRI results, a reliable characterization of the malignant nature of the mass was performed. Malignant transformation of endometriosis demonstrated hemorrhage and solid projections as enhancement on postcontrast T1WIs (9). The presence of an enhanced soft-tissue component within a blood-filled ovarian cystic mass was an evidence of malignant transformation. Dynamic contrast-enhanced MRI has been shown to be useful in differentiating malignancies from benign tumors (10). A previous study, however, has reported 
A

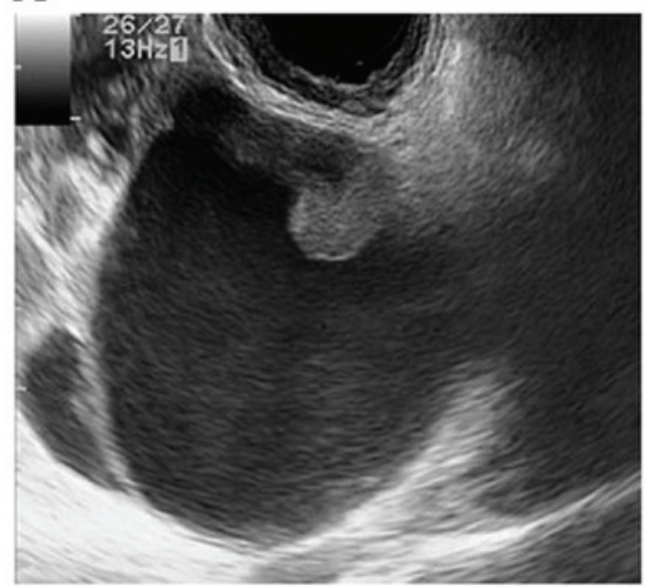

B

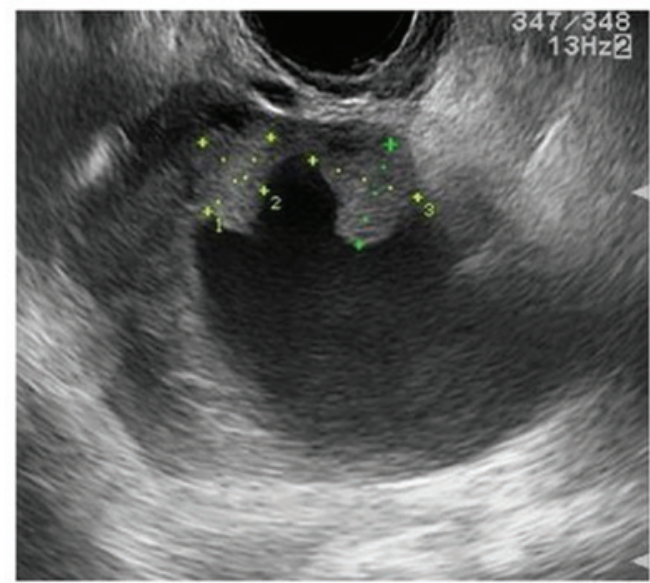

Figure 1. Transvaginal ultrasonography showed a $60-\mathrm{mm}$ multicystic mass with $12-\mathrm{mm}$ solid nodules. (A) Ultrasound patterns from the sagittal angle showed a bilocular cystic mass with a mural nodule. (B) Ultrasound patterns from a different angle showed two mural nodules with papillary projections.

A

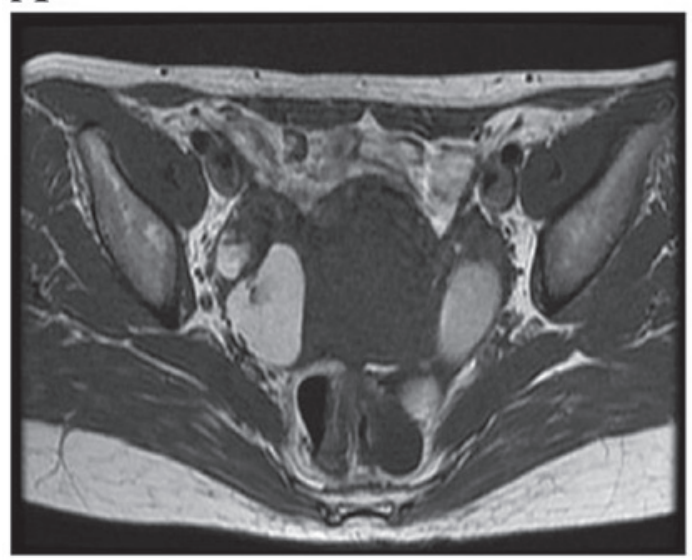

B

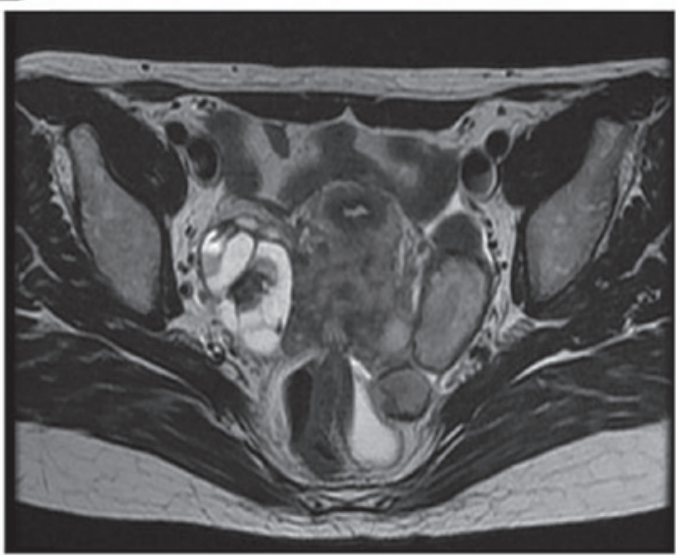

Figure 2. MRI results. Pelvic MRI showing high intensity on (A) T1-weighted images and (B) high-intensity with low-intensity spots on T2-weighted images. MRI, magnetic resonance imaging.

A

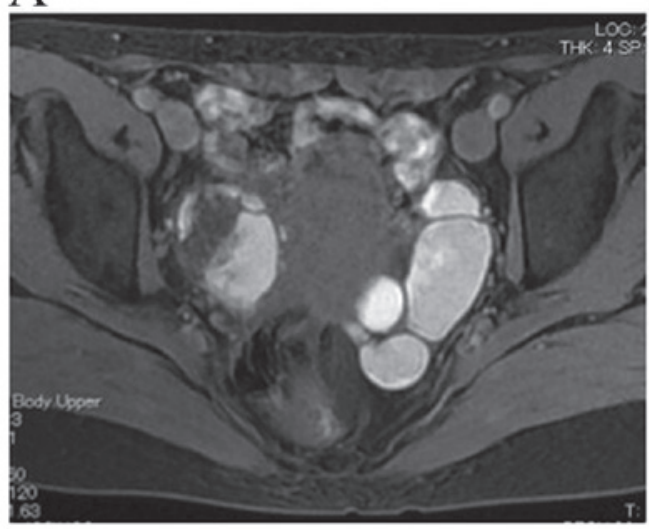

B

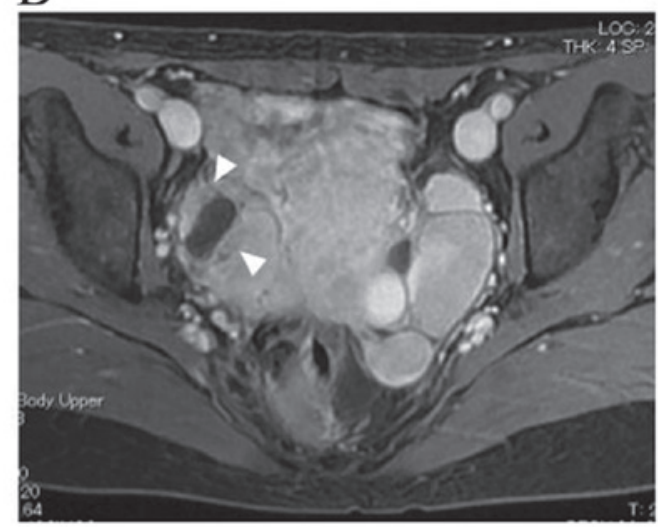

Figure 3. Dynamic contrast-enhanced, fat-suppressed, gradient-echo T1-weighted imaging was performed in the axial plane after an injection of gadolinium chelate components. Compared with (A) the mural nodules in the pre-contrast phase, (B) the mural nodules, indicated by arrowheads, were enhanced following the administration of the contrast medium.

that $21.4 \%$ of patients with benign OE have enhancing mural nodules, whose pathologies include atypical endometriosis, adenofibroma, fibrothecoma and papillary proliferation of the epithelium (4). Therefore, enhancement of the soft-tissue 


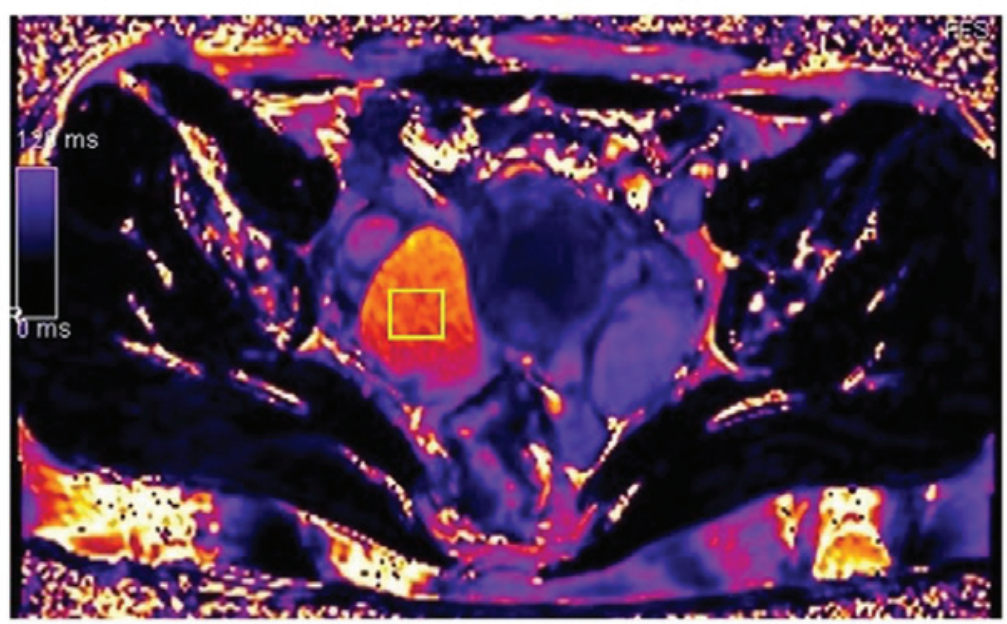

Figure 4. MR relaxometry results. MR relaxometry demonstrated a relaxation rate value of $7.98 \mathrm{~s}^{-1}$ in the right cyst, which suggested a malignant transformation of ovarian endometrioma. MR, Magnetic resonance.
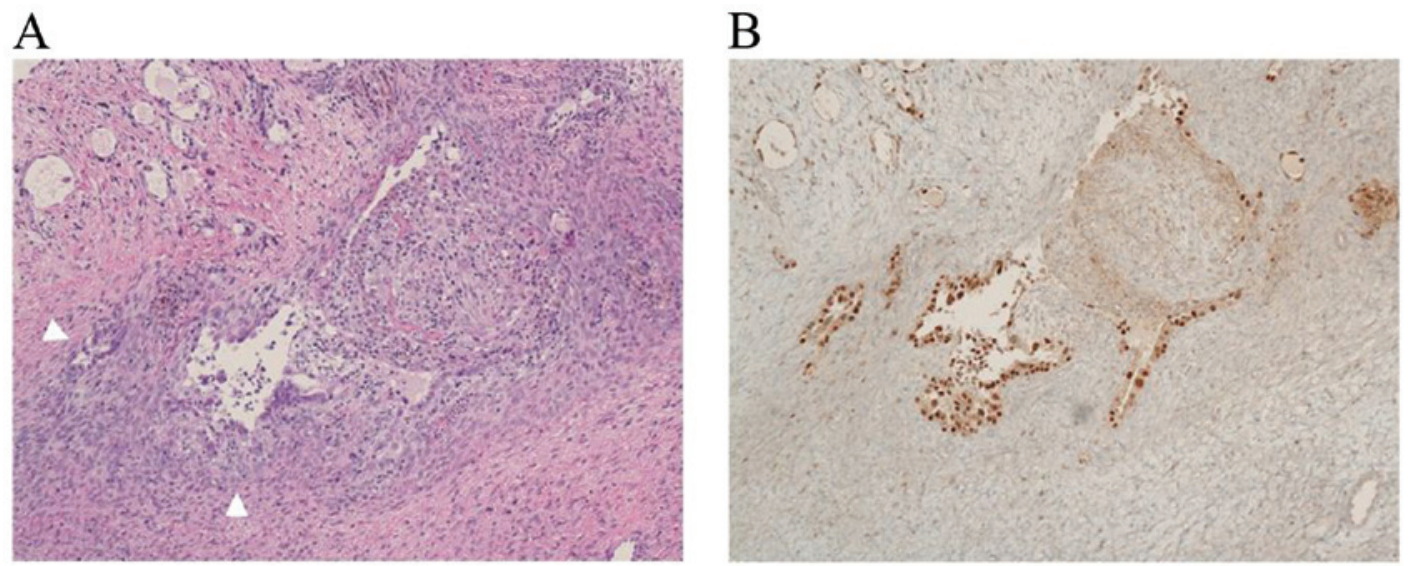

Figure 5. Histological results. (A) Cancer cells were localized within the cyst wall, indicated by arrowheads (magnification, x200). (B) Cells positive for hepatocyte nuclear factor-1 $\beta$ suggested clear cell carcinoma (magnification, $\mathrm{x} 200$ ).

elements on dynamic contrast-enhanced MRI would not always predict the diagnosis of ovarian malignancy.

In addition, ovarian cancer exhibits solid mural nodular lesions with enhancement after the intravenous administration of a contrast medium. Pre-treatment assessment by CT or MRI is helpful to identify patients who might benefit from neoadjuvant chemotherapy prior to surgical management. In contrast, a new imaging modality, MR relaxometry, can be used to qualitatively differentiate between benign and malignant lesions. MR relaxometry is a non-invasive, powerful tool for the direct detection of cystic fluid iron concentration. Yoshimoto et al (6) demonstrated that patients with EAOC had much lower levels of iron in the cystic fluid compared with patients with benign $\mathrm{OE}$, and iron concentration was a significant biomarker for the diagnosis of EAOC. An R2 value $<12.1 \mathrm{~s}^{-1}$ is predictive of malignancy (6). The $\mathrm{R} 2$ values exhibit excellent accuracy in distinguishing between EAOC and benign OE, with $86 \%$ sensitivity and $94 \%$ specificity (6). MR relaxometry can allow the distinction between benign $\mathrm{OE}$ with an enhancing soft-tissue component and EAOC. As malignant tissue was identified in a location different from the solid component found by MRI, diagnosis with MR relaxometry may present advantages compared with MRI. Therefore, MR relaxometry can facilitate the selection of patients who desire fertility-sparing surgery. Notably, in case of ovarian hemorrhage, an R2 value $<12.1 \mathrm{~s}^{-1}$ can be insufficient to diagnose ovarian cancer (data not shown). In addition, limitations may be present in case of multicenter collaborations.

In conclusion, conventional MRI and MR relaxometry were used to examine a case of clear cell carcinoma arising from $\mathrm{OE}$ where the patient desired fertility-preserving treatments. MR relaxometry may improve the accuracy and reduce the overdiagnosis of malignant transformations identified by MRI.

\section{Acknowledgements}

The authors would like to thank Dr Yuki Yamada (Department of Obstetrics and Gynecology, Nara Medical University) for technical assistance.

\section{Funding}

No funding was received. 


\section{Availability of data and materials}

The datasets used and analyzed during the current study are available from the corresponding author on reasonable request.

\section{Authors' contributions}

SM, DS, TW, AY, TM and MI collected, analyzed and interpreted the clinical data. AH, RM, NH and MM interpreted the pathological data. NK, CY and HK conceived the study. HK designed the study and interpreted clinical and pathological data. All authors read and approved the final version of the manuscript.

\section{Ethical approval and consent to participate}

The present study was approved by The Ethics Committee of the Nara Medical University.

\section{Patient consent for publication}

The patient signed a written informed consent.

\section{Competing interests}

The authors declare that they have no competing interests.

\section{References}

1. Kobayashi H, Sumimoto K, Kitanaka T, Yamada Y, Sado T, Sakata M, Yoshida S, Kawaguchi R, Kanayama S, Shigetomi $\mathrm{H}$, et al: Ovarian endometrioma-risks factors of ovarian cancer development. Eur J Obstet Gynecol Reprod Biol 138: 187-193, 2008.
2. Ogawa S, Kaku T, Amada S, Kobayashi H, Hirakawa T, Ariyoshi K, Kamura T and Nakano H: Ovarian endometriosis associated with ovarian carcinoma: A clinicopathological and immunohistochemical study. Gynecol Oncol 77: 298-304, 2000.

3. Park SB and Lee JB: MRI features of ovarian cystic lesions. J Magn Reson Imaging 40: 503-515, 2014.

4. Tanase Y, Kawaguchi R, Takahama J and Kobayashi H: Factors that differentiate between endometriosis-associated ovarian cancer and benign ovarian endometriosis with mural nodules. Magn Reson Med Sci 17: 231-237, 2018.

5. Lee S, Kim SK, Hwang KJ, Kim T and Kim SH: Fertility preservation for patients with gynecologic malignancies: The Korean Society for Fertility Preservation clinical guidelines. Clin Exp Reprod Med 44: 175-180, 2017.

6. Yoshimoto C, Takahama J, Iwabuchi T, Uchikoshi M, Shigetomi $\mathrm{H}$ and Kobayashi $\mathrm{H}$ : Transverse relaxation rate of cyst fluid can predict malignant transformation of ovarian endometriosis. Magn Reson Med Sci 16: 137-145, 2017.

7. Prat J and FIGO Committee on Gynecologic Oncology: FIGO's staging classification for cancer of the ovary, fallopian tube, and peritoneum: Abridged republication. J Gynecol Oncol 26: 87-89, 2015.

8. Kobayashi H: Screening, epidemiology, molecular biology, and treatment strategies for endometriosis-associated ovarian cancer. Reprod Med Biol 9: 17-22, 2009.

9. Takeuchi M, Matsuzaki K, Uehara H and Nishitani H: Malignant transformation of pelvic endometriosis: MR imaging findings and pathologic correlation. Radiographics 26: 407-417, 2006.

10. Li HM, Feng F, Qiang JW, Zhang GF, Zhao SH, Ma FH, Li YA and $\mathrm{Gu}$ WY: Quantitative dynamic contrast-enhanced MR imaging for differentiating benign, borderline, and malignant ovarian tumors. Abdom Radiol (NY) 43: 3132-3141, 2018. 